\title{
Lagrangian Embeddings in the Complement of Symplectic Hypersurfaces
}

\author{
Klaus Mohnke*
}

\begin{abstract}
For a given embedded Lagrangian in the complement of a complex hypersurface we show existence of a holomorphic disc in the complement having boundary on that Lagrangian.
\end{abstract}

\section{Introduction and Main Results}

Let $L \subset \mathbb{C}^{n}$ be a Lagrangian, i.e. immersed (real) $n$-manifolds $L \subset \mathbb{C}^{n}$ such that the restriction $\left.\omega\right|_{L}=0$ for the symplectic 2 -form $\omega=d \theta=\sum_{i=1}^{n} d x^{i} \wedge d y^{i}$ vanishes, where $\theta=\sum_{i=1}^{n} x^{i} d y^{i}$ is the Liouville form and $z^{i}=x^{i}+\mathrm{i} y^{i}$ are the (complex) coordinates on $\mathbb{C}^{n}$. Hence $\left.\theta\right|_{L}$ is a closed 1-form and thus defines an element in $\mathbf{H}^{\mathbf{1}}(L, \mathbb{Z})$. In [2] Gromov constructed non constant holomorphic discs with boundary on any embedded, closed Lagrangian in $\mathbb{C}^{n}$. He concluded that such a Lagrangian is never exact, i.e. $\left[\left.\theta\right|_{L}\right] \in \mathbf{H}^{\mathbf{1}}(L ; \mathbb{Z})$ defines a non vanishing class in de Rham cohomology: the symplectic volume of the disc is positive by the compatibility of complex and symplectic structure and so is the integral of $\theta$ over the boundary of the disc by Stokes' theorem. In this paper we specialize the disc found by Gromov in the presence of a complex hypersurface. Let us first fix some notation (see e.g. [1]).

Definition 1.1 A complete Riemannian manifold is called geometrically bounded (g.b.) if its sectional curvature is bounded above and the injectivity radius is bounded below. A submanifold is g.b. if its second fundamental form is bounded above and if there are constants $\epsilon, C>0$ such that if two points of the submanifold can be joined by a geodesic of length $l \leq \epsilon$ in the ambient space they can be joined by a geodesic of length $\mathrm{Cl}$ in the submanifold.

Note that if the manifold (submanifold) in question is compact it is, of course, geometrically bounded. Now we are ready to state the main statements of this note.

Theorem 1.2 (i) Suppose $L \cong \mathbb{R} \times \mathbf{S}^{\mathbf{1}}$ is a g.b. Lagrangian embedding into $\mathbb{C}^{2}$ such that there exists a complex line $H$ in its complement nontrivially linked by $L$, i.e. $0 \neq l k(H,):. \mathbf{H}_{\mathbf{1}}(L) \rightarrow \mathbb{Z}$. Then each complex linear function which is non-constant on $H$ is unbounded on $L$.

(ii) There is no Lagrangian embedding of the Klein bottle into the complement of a (real) plane in $\mathbb{C}^{2}$, nontrivially linking it.

Remark 1.3 (0) The Klein bottle is the only closed surface for which it is unknown wether it admits a Lagrangian embedding into $\mathbb{C}^{2}$ or not. For this some progress has been reported by Hofer and Luttinger [3].

(1) We may construct embedded totally real Klein bottles in the complement of a plane nontrivially

*Universität-Gesamthochschule Siegen, e-mail: mohnke@mathematik.uni-siegen.de 
linking it. Also note that there is an immersed Lagrangian Klein bottle in the complement of a plane linking it nontrivially (see Exercise 1.4.2. of [1])

(2) Lalonde proves in 4 that if there exists an embedded Lagrangian Klein bottle in $\mathbb{C}^{2}$ then the double points of the immersed one in (1) can be suppressed by a symplectic Whitney disc. It follows from Theorem 1.9 that this disc may never lie in the complement of the plane.

All this will follow from the main result of the paper. We will formulate it in a more general setting refining the arguments to specify the holomorphic disc to lie in the complement of a complex hypersurface with boundary on a given Lagrangian in that complement.

Theorem 1.4 Let $M^{2 n}$ be a symplectic manifold and $\Sigma \subset M$ be a symplectic hypersurface such that there exists a compatible almost complex structure such that $M$ and $\Sigma$ are geometrically bounded and there is no nonconstant holomorphic sphere in $M$ with nonpositive intersection index with the hypersurface $\Sigma$ inside $M$. Suppose $U \subset M$ is a neighbourhood of $\Sigma$ and $L \subset(M \backslash U) \times \mathbb{C}$ is a g.b. Lagrangian such that its projection onto the second component is bounded. Then there exists a loop $\gamma \subset L$ which bounds a nonconstant holomorphic disc in $(M \backslash \Sigma) \times \mathbb{C}$.

Proof of Theorem 1.2. (i) Assume there is a bounded complex linear function which is nonconstant on $H$ then the hypotheses of Theorem 1.4 are satisfied and in the complement of $H$ there is a disc with boundary on $L$ and nonvanishing symplectic area. However, this contradicts $1 \mathrm{k}(H,) \neq$. since the boundary of the disc represents an element of its kernel which is not torsion.

(ii) Let $L$ be a Lagrangian embedding of a closed surface into the complement of a (real) plane. Since for a plane to be symplectic is an open condition we may assume that the plane is symplectic (by replacing it with a nearby plane) and fix a constant, and thus integrable complex structure on $\mathbb{C}^{2}$ such that the plane is a complex line $H \subset \mathbb{C}^{2}$. In the complement of $H$ we find a disc with boundary on $L$ and nonvanishing symplectic area. Thus we obtain an element in the kernel of $\operatorname{lk}(H,):. \mathbf{H}_{\mathbf{1}}(L) \longrightarrow \mathbf{Z}$ which is not torsion. Hence $b_{1}(L) \geq 2$ and the statement follows.

There are also consequences in higher dimensions which are interesting from another point of view: Here we have much more space to construct Lagrangians of all kinds, for e.g. via a "cabeling" procedure (see Proposition 1.2.3 in 国). But the following corollary shows that not everything is possible:

Corollary 1.5 Let $L$ be a g.b. Lagrangian in the complement of a complex hyperplane $H \subset \mathbb{C}^{n}$. Then either

(i) each complex linear function on $\mathbb{C}^{n}$ is unbounded on $L$ or

(ii) the topology of $L$ is restricted by $b_{1}(L) \geq 1$

- if $L$ and the plane are unlinked or

- if each complex linear function which is bounded on $L$ vanishes on $H$

(iii) or $b_{2}(L) \geq 2$.

Proof. The hypotheses of Theorem 1.4 are satisfied. This, together with the assumptions on the linking and the existence of the complex linear function, implies the statement as in the proof of Theorem 1.2. If $L$ is closed we may take any real 4-plane by the perturbation argument of that proof.

Corollary 1.6 In particular, if $L$ is closed and nontrivially linked with any real $2(n-1)$-plane, then $b_{1}(L) \geq 2$. That means, for $n \geq 3$, that a Lagrangian embedding of $\mathbf{S}^{\mathbf{n}-\mathbf{1}} \times \mathbf{S}^{\mathbf{1}}$ in $\mathbb{C}^{n}$ (which e.g. can be constructed from Whitney's Lagrangian immersion of the sphere $\mathbf{S}^{\mathbf{n}-\mathbf{1}}$ into $\mathbb{C}^{n-1}$ via the cabling mentioned above) trivially links any real $2(n-1)$-plane in its complement. 


\section{Proof of Theorem 1.4}

We prove the observation refining the arguments used to prove the corresponding result of Gromov [2] (for more details see e.g. [1]).

Denote by $\pi: M \times \mathbb{C} \longrightarrow M$ the projection onto the first component. Fix an $\alpha>2$. We define

$$
\begin{aligned}
\mathcal{F} & :=\left\{u \in \mathbf{C}^{\alpha}((\Delta, \partial \Delta, 1),(M \times \mathbb{C}, L, p)) \mid u \cong p \text { in } \pi_{2}(M \times \mathbb{C}, L, p)\right\} \\
\mathcal{G} & :=\left\{g \in \mathbf{C}^{\alpha-1}\left(\Delta \times(M \times \mathbb{C}), T^{\mathbb{C}}(M \times \mathbb{C}) \mid \pi_{*}\left(\left.g\right|_{\Delta \times U \times \mathbb{C}}\right)=0\right\}\right. \\
\mathcal{M} & :=\{(u, g) \in \mathcal{F} \times \mathcal{G} \mid \bar{\partial} u-g\}
\end{aligned}
$$

where by $\bar{\partial}$ denotes the Cauchy-Riemann operator $\frac{1}{2}\left(\frac{\partial}{\partial x}+J \frac{\partial}{\partial y}\right)$ in complex coordinates $z=x+\mathrm{i} y$ on the disc.

The linearization

$$
D_{(u, g)}: \mathbf{C}^{\alpha}\left((\Delta, \partial \Delta, 1),\left(u^{*} T M \times \mathbb{C}, u^{*} T L, 0\right)\right) \times \mathcal{C}^{\alpha-1}\left(\Delta, u^{*} T M \times \mathbb{C}\right) \longrightarrow \mathcal{C}^{\alpha-1}\left(\Delta, u^{*} T M \times \mathbb{C}\right)
$$

of the equation $\bar{\partial} u=g$ is surjective for the following reason: there are sufficient degrees of freedom coming from the second component to perturb the equation at the boundary of the disc. Hence an element in the cokernel would satisfy the Cauchy-Riemann equation with Dirichlet conditions on the boundary which imply its vanishing. Thus

Proposition 2.1 $\mathcal{M} \subset \mathcal{F} \times \mathcal{G}$ is a Banach submanifold. The natural projection $P: \mathcal{M} \longrightarrow \mathcal{G}$ is a Fredholm map of index 0 . It is regular at $(p, 0)$.

Note that $P^{-1}(0)=\{(p, 0)\}$ consists of a single point. Pick $h:=(0, B)$ according to the splitting $T(M \times \mathbb{C}) \cong \pi^{*} T M \times \underline{\mathbb{C}}, \underline{\mathbb{C}}$ being the trivial complex line bundle, with $B \in \mathbb{C}$ understood as a constant section of it. Connect $h$ it to 0 by a path $\gamma \subset \mathcal{G}$ transversal w.r.t. $P$. Hence $P^{-1}(\gamma)$ is a one-dimensional manifold with boundary consisting of $(p, 0)$ and $P^{-1}(g)$. Via Cauchy-integral or Poisson-formula one proves that there is no solution to $\bar{\partial} u=h$ provide $B$ is sufficiently large. (see e.g. [1]). Therefore $P^{-1}(g)=\emptyset$. Thus $P^{-1}(\gamma)$ has to be non-compact.

Let $t_{0}:=\inf \left\{t \mid P^{-1}(\gamma(t))=\emptyset\right\}$. Pick a sequence $t_{n} \uparrow t_{0}>0$ and $\left(u_{n}, g_{n}\right) \in P^{-1}\left(\gamma\left(t_{n}\right)\right) \subset \mathcal{M}$ with $\lim _{n \rightarrow \infty} g_{n} \in g \in \mathcal{G}$ which does not converge in $\mathcal{M}$. From [2] (for a longer discussion see also [1]) we therefor know that there is "bubbling": a subsequence converges in the sense of Gromov to a solution of $\bar{\partial} u=g$ with $u:(\Delta, \partial \Delta) \longrightarrow(M \times \mathbb{C}, \Sigma \times \mathbb{C})$ representing a different homotopy class then the $u$ 's considered so far and a (nonempty!) collection of nonconstant holomorphic discs with boundary on $L$ and nonconstant holomorphic spheres counted with multiplicity. The convergence is such that the homology class of the $u_{n}$ is preserved. Hence the sum of intersection numbers of these holomorphic curves and $u$ with $\Sigma \times \mathbb{C}$ is zero. But the discs have non-negative intersection number with the hypersurface for they are holomorphic and the boundary is disjoint from it due to Lemma 2.3. Also the spheres have positive intersection number with $\Sigma \times \mathbb{C}$ by assumption.

Thus it suffices to show that $u$ has nonnegative intersection number with $\Sigma \times \mathbb{C}$. For this excludes spheres in the collection of holomorphic curves. Hence there is a nonconstant holomorphic disc with boundary on $L$ and zero intersection index with $\Sigma \times \mathbb{C}$ which implies that it has to lie in the complement.

Let us prove the statement about $u$. Assume the contrary. Pick a point $q \in u(\Delta) \cap \Sigma \times \mathbb{C}$. We claim that its intersection index is positive. Since $\pi_{*}\left(\left.g\right|_{\Delta \times U \times \mathbb{C}}\right)=0$ we obtain a holomorphic map $\pi \circ u: u^{-1}(U \times \mathbb{C}) \subset \Delta \rightarrow M$ near the preimage of $q$. The intersection indices of $u$ with $\Sigma \times \mathbb{C}$ at $q$ and $\pi \circ u$ with $\Sigma$ at $\pi(q)$ agree. Due to Lemma 2.3 the latter is a well-defined (finite!) positive number unless the whole image of that neighbourhood lies inside $\Sigma$. This cannot happen because then $u$ would map completely into $\Sigma \times \mathbb{C}$. But at least the boundary lies in $L \subset(M \backslash \Sigma) \times \mathbb{C}$. 
Remark 2.2 Theorem 1.9 has a simpler proof directly applying Gromov's original theorem using the fact that $\mathbb{C}^{n} \backslash \mathbb{C}^{n-1}$ can be symplectomorhically embedded in $\left(\mathbb{R} \times \mathbf{S}^{\mathbf{1}}\right) \times \mathbb{C}^{n-1}$

This situation belongs to the case when the complement of $\Sigma$ is Stein. Here one may argue with the non-existence of holomorphic curves touching some (weakly) pseudoconvex boundary from the inside instead. However, the statement is true in a much more general context.

Let us finish with a discussion on the positiveness of the intersection indices of an embedded almost complex hypersurface and a (pseudo)holomorphic curve not lying inside the former.

Lemma 2.3 Consider an almost comlex manifold $M$. Let $\Sigma \subset M$ be an embedded almost complex hypersurface and $u: \Delta \longrightarrow M$ be a (pseudo)holomorphic curve. Then either the image of $u$ is completely contained inside $\Sigma$ or $u^{-1}(\Sigma) \subset \Delta$ is a discrete set inside the open disc $\Delta$ each point coming with a finite intersection index.

Proof. The notion of the index will become clear during the discussion of the proof. The following argument is due to Brian White. Near an intersection point we may pick coordinates such that $M$ looks like a subset of $\mathbb{C}^{n-1} \times \mathbb{C}$ (containing 0 say) and the almost complex structur has the form $J(z, \zeta)=\left(\begin{array}{cc}j(z, \zeta) & 0 \\ O(\zeta)(z, \zeta) & i\end{array}\right)$ where $j$ is an almost complex structure on $\mathbb{C}^{n-1}, i$ is the ordinary complex multiplication and $O(\zeta)$ is a smooth $2(n-1) \times 2$-matrix-valued function (with real entries) vanishing of first order with $|\zeta|$ satisfying $O(\zeta) j+i O(\zeta)=0$. Now, if $(U, u): \Delta \longrightarrow \mathbb{C}^{n-1} \times \mathbb{C}$ is a pseudo-holomorphic curve through $(0,0)$ then in the coordinate chart it satisfies the following differential equation

$$
\frac{\partial u}{\partial \bar{z}}=O^{\prime}(u) D U
$$

again with a matrix-valued function $O^{\prime}(\zeta)$ of the kind above. Differentiating this equation we end up with the differential inequality

$$
|\Delta u| \leq K(|u|+|D u|)
$$

for the $u$-part of that pseudoholomorphic curve. As in [5] we conclude that either the whole image of the curve will be contained in the hypersurface or there is a positive integer $m$ such that $u(\xi)=\xi^{m}+O\left(|\xi|^{m+1}\right)$ proving the statement of the lemma. Another argument was given by Jean-Claude Sikorav. He concludes the result using similarity principle at the first order differential equation for $u$ as in his paper [6].

Acknowledgements. I would like to thank Yakov Eliashberg for the initial discussions on this subject, Leonid Polterovich for his cordial and patient help with the paper, Brian White and Jean-Claude Sikorav for the help with the proof of Lemma 2.3.

\section{References}

[1] Michéle Audin, François Lalonde, and Leonid Polterovich. Symplectic rigidity: Lagrangian submanifolds. In Holomorphic curves in symplectic geometry, volume 117 of Progress in Math., pages 271-321. Birkhäuser Verlag, 1994.

[2] Michail Gromov. Pseudo-holomorphic curves in symplectic manifolds. Invent. math., 82:307$347,1985$.

[3] Helmut Hofer and Karl Luttinger. 1996.

[4] Françoise Lalonde. Suppression lagrangienne de points doubles et rigidité symplectique. J. Diff. Geom., 36:747-764, 1992. 
[5] Mario J. Micallef and Brian White. The structure of branch points in minimal surfaces and in pseudoholomorphic curves. Annals of Mathematics, 139:35-85, 1994.

[6] Jean-Claude Sikorav. Singularities of J-holomorphic curves. Math. Zeitschr., 226:359-373, 1997. 